\title{
A Rational Approximation to the Logarithm
}

\author{
By R. P. Kelisky and T. J. Rivlin
}

We shall use the $\tau$-method of Lanczos to obtain rational approximations to the logarithm which converge in the entire complex plane slit along the nonpositive real axis, uniformly in certain closed regions in the slit plane. We also provide error estimates valid in these regions.

1. Suppose $z$ is a complex number, and $s(1, z)$ denotes the closed line segment joining 1 and $z$. Let $y$ be a positive real number, $y \neq 1$. As a polynomial approximation, of degree $n$, to $\log x$ on $s(1, y)$ we propose $p^{*} \in P_{n}, P_{n}$ being the set of polynomials with real coefficients of degree at most $n$, which minimizes

$$
\left\|x p^{\prime}(x)-1\right\|=\max _{x \in s(1, y)}\left|x p^{\prime}(x)-1\right|
$$

among all $p \in P_{n}$ which satisfy $p(1)=0$. (This procedure is suggested by the observation that $w=\log x$ satisfies $x w^{\prime}(x)-1=0, w(1)=0$.)

We determine $p^{*}$ as follows. Suppose $p(x)=a_{0}+a_{1} x+\cdots+a_{n} x^{n}, p(1)=0$ and

$$
x p^{\prime}(x)-1=\pi(x)=b_{0}+b_{1} x+\cdots+b_{n} x^{n}
$$

Then

$$
\begin{aligned}
& b_{0}=-1, \\
& a_{j}=b_{j} / j, \quad j=1, \cdots, n,
\end{aligned}
$$

and

$$
a_{0}=-\sum_{j=1}^{n} a_{j}
$$

Thus, minimizing $\left\|x p^{\prime}-1\right\|$ subject to $p(1)=0$ is equivalent to minimizing $\|\pi\|$ among all $\pi \in P_{n}$ which satisfy $-\pi(0)=1$. This, in turn, is equivalent to maximizing $|\pi(0)|$ among all $\pi \in P_{n}$ which satisfy $\|\pi\|=1$, in the sense that if $\pi_{0}$ maximizes $|\pi(0)|$ subject to $\|\pi\|=1$, then $\pi^{*}=-\pi_{0} / \pi_{0}(0)$ minimizes $\|\pi\|$ subject to $-\pi(0)=$ 1. For, $-\pi^{*}(0)=1$ and since $\left\|\pi_{0}\right\|=1,\left\|\pi^{*}\right\|=1 /\left|\pi_{0}(0)\right|$. Now suppose $\pi \in P_{n}$ satisfies $-\pi(0)=1$, then $\pi_{1}=\pi /\|\pi\|$ has norm 1 and so $\left|\pi_{1}(0)\right|=|\pi(0)| /\|\pi\|=$ $1 /\|\pi\| \leqq\left|\pi_{0}(0)\right|=1 /\left\|\pi^{*}\right\|$, from which we conclude that $\left\|\pi^{*}\right\| \leqq\|\pi\|$.

Since 0 is exterior to $s(1, y)$, it is known (cf. Rivlin and Shapiro [3, p. 684]) that the Chebyshev polynomial for $s(1, y)$,

$$
T_{n, y}(x)=T_{n}((2 x-(1+y)) /(1-y)),
$$

(where $T_{n}(x)=\cos n \theta, x=\cos \theta, 0 \leqq \theta \leqq \pi$ ) is the polynomial satisfying $\left\|T_{n, y}\right\|=1$ and $|\pi(0)|<\left|T_{n, y}(0)\right|$ for all $\pi \in P_{n},\|\pi\|=1, \pi \neq \pm T_{n, y}$. Thus $\pi_{0}$ is $T_{n, y}$ and $\left\|x p^{\prime}-1\right\|$ is minimized, subject to $p(1)=0$ for $p=p^{*}$ when

Received February 6, 1967. Revised May 18, 1967. 


$$
x p^{* \prime}(x)-1=\pi^{*}(x)=T_{n, y}(x) /-T_{n, y}(0),
$$

and if we put $p=p^{*}$ and $\pi(x)=T_{n, y}(x) /\left(-T_{n, y}(0)\right)$ in (1), then the coefficients of $p^{*}, a_{0}, \cdots, a_{n}$, are easily determined by (2), (3), and (4). We obtain explicitly

$$
\begin{aligned}
& a_{j}=\frac{(-1)^{j+1} 2^{j}}{j T_{n}((y+1) /(y-1))(1+y)^{j}} \sum_{i=j}^{n}(-1)^{i}\left(\begin{array}{l}
i \\
j
\end{array}\right)\left(\frac{1+y}{1-y}\right)^{i} t_{i}, \\
& i=1, \cdots, n,
\end{aligned}
$$

and

$$
a_{0}=-\left(a_{1}+\cdots+a_{n}\right),
$$

where $t_{i}$ is the coefficient of $x^{i}$ in $T_{n}(x)$. Thus, $a_{0}, \cdots, a_{n}$ are rational functions of $y$, and so is

$$
r_{n}(y)=p^{*}(y)=a_{0}(y)+a_{1}(y) y+\cdots+a_{n}(y) y^{n} .
$$

We shall take $r_{n}(z)$, where $z$ is any complex number which does not satisfy $z \leqq 0$, as our rational approximation to $\log z$, and study its convergence to $\log z$ as $n \rightarrow \infty$ in the next section. The determination of the polynomial approximation to $\log x$, $p^{*}(x)$, by means of (5) is Lanczos' $\tau$-method with $\tau=-1 / T_{n, y}(0)$. Lanczos [2] starts with $\tau T_{n, y}$ on the right-hand side in (5), whereas we have provided here a justification of this choice. A more detailed and systematic exposition of this view of the $\tau$ method is given in Rivlin and Weiss [4].

2. The rational function defined in (8) can be rewritten in the form

$$
r_{n}(z)=\frac{\sum_{j=1}^{n}(-1)^{j+1} \frac{2^{j}\left(z^{j}-1\right)}{j} \sum_{i=j}^{n}(-1)^{i}\left(\begin{array}{l}
i \\
j
\end{array}\right) t_{i}(1+z)^{i-j}(1-z)^{n-i}}{\sum_{j=0}^{n}(-1)^{j} t_{j}(1+z)^{j}(1-z)^{n-j}}
$$

which reveals that the numerator and denominator of $r_{n}(z)$ are polynomials of degree $n$. Before studying the properties of $r_{n}(z)$ further, we wish to consider its effectiveness as an approximation to $\log z$.

Suppose that $z \neq 1$ is a complex number which does not satisfy $z \leqq 0$. Then if $w \in s(1, z)$ we integrate the differential equation

$$
w p^{* \prime}(w)-1=T_{n, z}(w) /-T_{n, z}(0)
$$

along $s(1, z)$ to obtain, after an elementary change of variables,

$$
r_{n}(z)-\log z=\frac{1}{T_{n}((z+1) /(z-1))} \int_{-1}^{1} \frac{T_{n}(t)}{t-((z+1) /(z-1))} d t,
$$

where we have used the facts that $p^{*}(1)=0, p^{*}(z)=r_{n}(z)$ and $T_{n, z}(0)=$ $T_{n}((z+1) /(z-1))$.

Note that the point

$$
\zeta=(z+1) /(z-1)
$$

is exterior to $[-1,1]$. Let $C_{\rho}$, for $0<\rho<1$, denote the ellipse in the $\zeta$-plane, $\zeta=\xi+i \eta$ 


$$
\begin{aligned}
& \xi=\frac{1}{2}(\rho+(1 / \rho)) \cos \phi, \\
& \eta=\frac{1}{2}(\rho-(1 / \rho)) \sin \phi,
\end{aligned}
$$

which has foci at $( \pm 1,0), \rho$ as sum of its semiaxes and passes through the point $\zeta$. Thus

$$
(\rho+(1 / \rho)) / 2=(1+|z|) /|z-1| .
$$

For some $\phi_{0}, 0 \leqq \phi_{0}<2 \pi$ we have

$$
\begin{aligned}
T_{n}(\zeta) & =\frac{1}{2}\left\{\left(\zeta+\left(\zeta^{2}-1\right)^{1 / 2}\right)^{n}+\left(\zeta-\left(\zeta^{2}-1\right)^{1 / 2}\right)^{n}\right\} \\
& =\frac{1}{2}\left\{\rho^{n} \exp \left(i n \phi_{0}\right)+\rho^{-n} \exp \left(-i n \phi_{0}\right)\right\}
\end{aligned}
$$

and so

$$
1 /\left|T_{n}(\zeta)\right| \leqq 2 /\left(\rho^{-n}-\rho^{n}\right)
$$

Lemma 1. If $-1 \leqq x \leqq 1$ and

$$
S_{n}(x)=\int_{-1}^{x} T_{n}(t) d t
$$

then

$$
\begin{aligned}
\left|S_{n}(x)\right| & \leqq \frac{1}{2}, & & n=1 \\
& \leqq 1 /(n-1), & & n>1
\end{aligned}
$$

and

$$
\begin{aligned}
S_{n}(1) & =0, \quad n \text { odd }, \\
& =-2 /\left(n^{2}-1\right), \quad n \text { even } .
\end{aligned}
$$

The proof is by direct computation and we omit it.

LEMMA 2.

$$
\left|\int_{-1}^{1} \frac{T_{n}(t)}{t-\zeta} d t\right| \leqq \frac{2}{\left(n^{2}-1\right)|1-\zeta|}+\frac{1}{n-1} \int_{-1}^{1} \frac{d t}{|t-\zeta|^{2}}, \quad n>1 .
$$

Proof. Integration by parts yields

$$
\int_{-1}^{1} \frac{T_{n}(t)}{t-\zeta} d t=\frac{S_{n}(1)}{1-\zeta}+\int_{-1}^{1} \frac{S_{n}(t)}{(t-\zeta)^{2}} d t
$$

and the conclusion follows from Lemma 1.

LEMMA 3.

$$
\int_{-1}^{1} \frac{d t}{|t-\zeta|^{2}} \leqq \frac{8}{[\rho+(1 / \rho)-2]^{2}}
$$

Proof. (See Fig. 1.)

Consider Fig. 1. Suppose $\zeta$ is located on the arc of $C_{\rho}$ in the first quadrant. Certainly $|\zeta-t| \geqq \operatorname{Im} \zeta$ for real $t$, hence if $\operatorname{Re} \zeta \leqq 1,|\zeta-\mathrm{t}| \geqq \operatorname{Im} \zeta \geqq Q F$. However, since $F^{\prime} P+P F=$ const. for any point $P$ of the ellipse, we conclude that $Q F \geqq$ $A F$. Also, if $\operatorname{Re} \zeta>1$, then $|\zeta-\mathrm{t}| \geqq|\zeta-1| \geqq A F$. But

$$
A F=(\rho+(1 / \rho)-2) / 2 \text {. }
$$




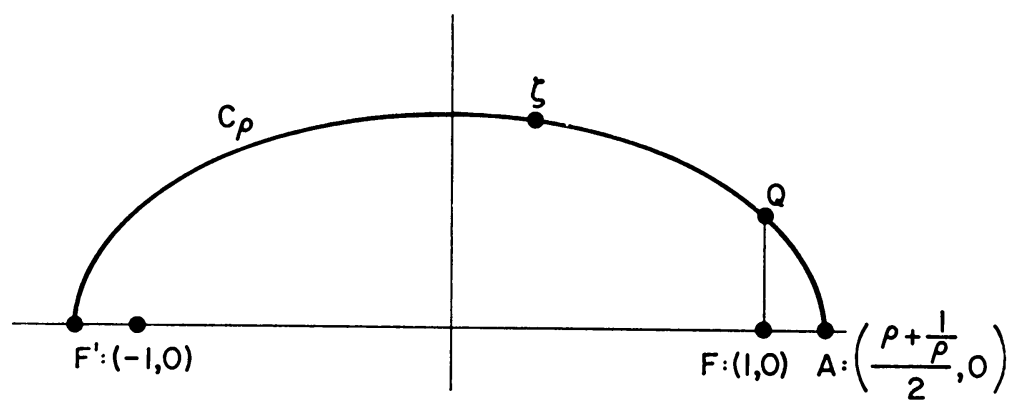

Figure 1

Hence

$$
1 /|t-\zeta|^{2} \leqq 4 /(\rho+(1 / \rho)-2)^{2}, \quad-1 \leqq t \leqq 1
$$

Considerations of symmetry show that the same bound holds for any position of $\zeta$ on $C_{\rho}$, and the conclusion of the lemma follows.

We can now prove

Theorem 1. If $z$ does not satisfy $z \leqq 0$, then for $n>1$

$$
\left|r_{n}(z)-\log z\right| \leqq c(\rho) \rho^{n} / n,
$$

where $\rho$ is the unique solution of (12) which satisfies $0 \leqq \rho<1$ (the value $\rho=0$ corresponding to $z=1$ ) and $c(\rho)$ is a finite quantity depending only on $\rho$.

Proof. In view of (10), (11), (13) and Lemmas 2 and 3, we obtain

$$
\left|r_{n}(z)-\log z\right| \leqq \frac{2}{\rho^{-n}-\rho^{n}}\left[\frac{2}{\left(n^{2}-1\right)|1-\zeta|}+\frac{8}{(n-1)[\rho+(1 / \rho)-2]^{2}}\right] .
$$

As we saw in the proof of Lemma 3

$$
|1-\zeta| \geqq(\rho+(1 / \rho)-2) / 2,
$$

and so

$$
\begin{aligned}
\left|r_{n}(z)-\log z\right| & \leqq \frac{\rho^{n}}{n-1}\left(\frac{8}{1-\rho^{2 n}}\right)\left(\frac{1}{\rho+(1 / \rho)-2}\right)\left(\frac{1}{n+1}+\frac{2}{\rho+(1 / \rho)-2}\right) \\
& \leqq\left(\frac{8}{1-\rho^{4}}\right)\left(\frac{1}{\rho+(1 / \rho)-2}\right)\left(1+\frac{4}{\rho+(1 / \rho)-2}\right) \frac{\rho^{n}}{n} .
\end{aligned}
$$

The theorem follows by choosing

$$
c(\rho)=\frac{8}{1-\rho^{4}}\left(\frac{1}{\rho+(1 / \rho)-2}\right)^{2}\left(\rho+\frac{1}{\rho}+2\right) .
$$

Remark 1. The choice of $c(\rho)$ made in (15) is larger than necessary. It is clear, for example, that numbers $c(\rho, n)$ that have the property

$$
c(\rho, n) \sim 16 /(\rho+(1 / \rho)-2)^{2}, \quad n \rightarrow \infty,
$$

will serve as well in (14). 
Remark 2. If $n=1$

$$
\left|r_{1}(z)-\log z\right| \leqq 8 \rho /\left(1-\rho^{2}\right)(\rho+(1 / \rho)-2)^{2} .
$$

Remark 3. If $z>1$ then Theorem 1 holds with

$$
c(\rho)=c(\rho, n) \sim 16 /\left((\rho+(1 / \rho))^{2}-4\right), \quad n \rightarrow \infty,
$$

as a reexamination of the proof reveals.

Theorem 1 establishes the convergence of our rational approximation to the logarithm at each point of the slit plane. The bound obtained in (14) holds for all $z$ whose images in the $\zeta$-plane under the mapping (11) lie on $C_{\rho}$. It is not difficult to verify that these points form the limaçon, $P_{\rho}, 0<\rho<1$, defined by

$$
z=1+\frac{4 \rho}{1-\rho^{2}}\left(\frac{1+\rho^{2}}{1-\rho^{2}}+\frac{2 \rho}{1-\rho^{2}} \cos \theta\right) e^{i \theta}, \quad-\pi \leqq \theta<\pi,
$$

or, if we introduce polar coordinates $(R, \theta)$ in the $z$-plane, with pole at $z=1, P_{\rho}$ is given by

$$
R=4 \rho\left(1+\rho^{2}+2 \rho \cos \theta\right) /\left(1-\rho^{2}\right)^{2} .
$$

This is perhaps most readily seen by noting that the sequence of mappings

$$
z=\lambda^{2}, \quad \lambda=(w+1) /(w-1), \quad \zeta=\left(w+w^{-1}\right) / 2
$$

is equivalent to the mapping (11). $C_{\rho}$ is the image of $|w|=\rho$ under $\zeta=\left(w+w^{-1}\right) / 2$, and if $K_{\rho}$ is the circle in the $\lambda$-plane which is the image of $|w|=\rho$ under $\lambda=$ $(w+1) /(w-1)$ then $P_{\rho}$ is the image of $K_{\rho}$ under the mapping $z=\lambda^{2}$. From Kober, Dictionary of Conformal Representations, Dover, New York, 1952, p. 38, we find that $P_{\rho}$ is given by (16). Let $\dot{P}_{\rho}$ denote the closed set in the $z$-plane consisting of $P_{\rho}$ and all points inside the curve $P_{\rho}$. Since, under the mapping (11) $\dot{P}_{\rho}$ corresponds to $C_{\rho}$ plus its exterior (in a 1-1 conformal fashion), $\dot{P}_{\rho}$ is a simply-connected closed region which does not meet $z \leqq 0$. Therefore, we have

Theorem 2. If $z \in \dot{P}_{\rho}, 0<\rho<1$, then for $n>1$

$$
\left|r_{n}(z)-\log z\right| \leqq c(\rho) \rho^{n} / n,
$$

where $c(\rho)$ may be defined by (15).

Proof. From (9) we conclude that $r_{n}(z)$ has poles only at the zeros of $T_{n}((z+1) /(z-1))$, which are the points of the negative real axis

$$
z_{j}=\left(\zeta_{j}+1\right) /\left(\zeta_{j}-1\right)
$$

where

$$
\zeta_{j}=\cos ((2 j-1) \pi / 2 n), \quad j=1, \cdots, n .
$$

Therefore $r_{n}(z)-\log z$ is a single-valued analytic function in $\dot{P}_{\rho}$, and since (18) holds on $P_{\rho}$, it holds throughout $\dot{P}_{\rho}$ by the maximum modulus principle.

The set of limaçon domains $\dot{P}_{\rho}, 0<\rho<1$, satisfy $\dot{P}_{\rho_{1}} \subset \dot{P}_{\rho_{2}}$ when $\rho_{1}<\rho_{2}$, and every $z$ not satisfying $z \leqq 0$ is contained in $\dot{P}_{\rho}$ for $\rho$ close enough to 1 . A typical $\mathrm{P}_{\rho}$, $\rho=.5$, is shown in Fig. 2. 




Figure 2

3. Having established the convergence of our rational approximations, we wish to examine other of their properties. The function $\log z$ satisfies the functional equation $\log z=-\log (1 / z)$. We wish to show that the same is true of $r_{n}(z)$.

THEOREM 3. $r_{n}(z)$ as defined in (9) satisfies the functional equation

$$
r_{n}(z)=-r_{n}(1 / z)
$$

for all $z$ not satisfying $z \leqq 0$.

Proof. Since $t_{j}$ is the coefficient of $x^{j}$ in $T_{n}(x), t_{j}=0$ for $j \neq n-2 i, i=0, \cdots$, $[n / 2]$ and thus, for $j=0, \cdots, n$,

$$
(-1)^{j} t_{j}=(-1)^{n} t_{j} .
$$

(19) now follows easily upon replacing $z$ by $1 / z$ in (9).

Remark 1. In the slit plane, $-\pi<\arg z<\pi, f(z)=\log z$ also satisfies $f(\bar{z})=$ $[f(z)]^{*}$ and so does $f(z)=r_{n}(z)$. The asterisk denotes the complex conjugate in the preceding sentence.

Remark 2. The limaçon $P_{\rho}$ defined in (16) is transformed onto itself by the mappings $z \rightarrow \bar{z}, z \rightarrow 1 / \bar{z}$, hence also by $z \rightarrow 1 / z$, and the same is true of $\dot{P}_{\rho}$. Thus, approximation to $\log z$ throughout $\dot{P}_{\rho}$ is easily obtainable from evaluations of $r_{n}(z)$ in $\dot{P}_{\rho} \cap$ $\{|z| \leqq 1\}$.

By utilizing (20) we rewrite (9) as

$$
r_{n}(z)=N_{n}(z) / D_{n}(z)
$$

where 


$$
\begin{aligned}
& N_{n}(z)=\sum_{j=1}^{n}(-1)^{j+1} \frac{2^{j}\left(z^{j}-1\right)}{j} \sum_{i=j}^{n}\left(\begin{array}{l}
i \\
j
\end{array}\right) t_{i}(1+z)^{i-j}(1-z)^{n-i} \\
& D_{n}(z)=\sum_{j=0}^{n} t_{j}(1+z)^{j}(1-z)^{n-j} .
\end{aligned}
$$

Theorem 4.

$$
D_{n}(z)=\sum_{j=0}^{n}\left(\begin{array}{c}
2 n \\
2 j
\end{array}\right) z^{j} ; \quad n=1,2, \cdots
$$

and

$$
D_{n}(z)=2(1+z) D_{n-1}(z)-(1-z)^{2} D_{n-2}(z), \quad n=2,3, \cdots .
$$

Proof. According to (23)

$$
D_{n}(z)=(1-z)^{n} T_{n}((1+z) /(1-z)) .
$$

But

$$
T_{n}(w)=\frac{\left(w+\left(w^{2}-1\right)^{1 / 2}\right)^{n}+\left(w-\left(w^{2}-1\right)^{1 / 2}\right)^{n}}{2}
$$

so that

$$
D_{n}(z)=\frac{1}{2}\left[(1+\sqrt{ } z)^{2 n}+(1-\sqrt{ } z)^{2 n}\right]
$$

and (24) follows at once. Equation (25) follows from (26) and the three-term recurrence relationship for the Chebyshev polynomials.

Remark. We observe from (24) that the coefficients of $D_{n}$ are palindromic. Moreover, it is clear from (22) that $z-1$ is a factor of $N_{n}(z)$ and that $z^{n} N_{n}(1 / z)=-N_{n}(z)$ so that $N_{n}(z)=(z-1) \tilde{N}_{n-1}(z)$.

These two relations imply that the coefficients of $\tilde{N}_{n-1}$ are palindromic.

THEOREM 5. The numerator of $r_{n}(z)$ satisfies

$$
\begin{aligned}
& N_{n}(z)=-(1-z)^{n} \int_{-1}^{1} \frac{T_{n}(t)-T_{n}(\lambda)}{t-\lambda} d t \\
& N_{n}(z)=2(z-1) \sum_{k=0 ; k \text { even }}^{n-1} \frac{2}{k+1}(1-z)^{k} D_{n-1-k}(z)
\end{aligned}
$$

and the recurrence relationship

$$
N_{n}(z)=2(1+z) N_{n-1}(z)-(1-z)^{2} N_{n-2}(z)-2(1-z)^{n} \phi_{n},
$$

where

$$
\lambda=(1+z) /(1-z), \quad \sum_{k=0}^{m} u_{k}=u_{0}+u_{1}+\cdots+u_{m-1}+u_{m} / 2,
$$

and

$$
\begin{aligned}
\phi_{n} & =0, \quad n \text { even }, \\
& =-2 / n(n-2), \quad n \text { odd } .
\end{aligned}
$$

Proof. If we write $r_{n}(z)=N_{n}(z) / D_{n}(z)$, and observe that 


$$
\log z=\int_{-1}^{1} \frac{1}{t-\lambda} d t
$$

then (27) follows from

$$
\log z-r_{n}(z)=\frac{1}{T_{n}(\lambda)} \int_{-1}^{1} \frac{T_{n}(t)}{t-\lambda} d t
$$

which is simply (10), with $z$ replaced by $1 / z$, keeping (19) and (26) in mind.

In order to verify (28) we use the generating functions

$$
\begin{aligned}
\sum_{j=0}^{\infty} T_{j}(t) u^{j} & =\frac{1-u t}{1-2 t u+u^{2}}=\frac{1-u t}{\Delta(t)}, \\
1+2 \sum_{j=1}^{\infty} T_{j}(t) u^{j} & =\frac{1-u^{2}}{\Delta(t)},
\end{aligned}
$$

and

$$
\sum_{j=0}^{\infty} \frac{T_{j+1}^{\prime}(t)}{j+1} u^{j}=\frac{1}{\Delta(t)}
$$

Now

$$
\frac{1}{t-\lambda}\left[\frac{1-u t}{\Delta(t)}-\frac{1-u \lambda}{\Delta(\lambda)}\right]=\left[\frac{u}{\Delta(t)}\right]\left[\frac{1-u^{2}}{\Delta(\lambda)}\right],
$$

from which it is easy to see that

$$
\begin{aligned}
\int_{-1}^{1} \frac{T_{n}(t)-T_{n}(\lambda)}{t-\Lambda} d t & =\int_{-1}^{1}\left[\sum_{k=0}^{n-1} 2 T_{n-1-k}(\lambda) \frac{T_{k+1}^{\prime}(t)}{k+1}\right] d t \\
& =4 \sum_{k=0 ; k \text { even }}^{n-1} \frac{T_{n-1-k}(\lambda)}{k+1} .
\end{aligned}
$$

Thus, we conclude from (27) that

$$
N_{n}(z)=-4(1-z)^{n} \sum_{k=0 ; k \text { even }}^{n-1} \frac{T_{n-1-k}(\lambda)}{k+1}
$$

and (28) now follows from (26) and the definition of $\lambda$.

(29) is a consequence of (27) and the three-term recurrence relationship for the Chebyshev polynomials.

Remark. To find the value of $r_{n}(z)=N_{n}(z) / D_{n}(z)$, one can either utilize the two (closely related) recurrence relationships (29) and (25), or take advantage of the fact that in the form (28) $N_{n}(z)$ is expressed in terms of $D_{0}, \cdots, D_{n-1}$.

The first few approximations are the following:

$$
\begin{aligned}
& r_{1}(z)=2 \frac{z-1}{z+1} ; \quad r_{2}(z)=4 \frac{(z-1)(1+z)}{1+6 z+z^{2}} \\
& r_{3}(z)=\frac{2}{3} \frac{(z-1)\left(7+34 z+7 z^{2}\right)}{\left(1+15 z+15 z^{2}+z^{3}\right)} ; \quad r_{4}(z)=\frac{16}{3} \frac{(z-1)\left(1+11 z+11 z^{2}+z^{3}\right)}{\left(1+28 z+70 z^{2}+28 z^{3}+z^{4}\right)} \\
& r_{5}(z)=\frac{2}{15} \frac{(z-1)\left(43+868 z+2018 z^{2}+868 z^{3}+43 z^{4}\right)}{\left(1+45 z+210 z^{2}+210 z^{3}+45 z^{4}+z^{5}\right)}
\end{aligned}
$$


The form of these approximations resembles those obtained from the continued fraction expansion of Euler (cf. Khovanskii [1, Formula 4.7, p. 112]), but the actual coefficients appearing are different for $n>1$. Indeed, it appears that the Euler approximations are obtainable from ours by replacing the Chebyshev polynomials throughout by Legendre polynomials, which suggests that the convergents in the Euler expansion would be obtained by replacing the uniform norm by the leastsquares norm in the minimum problem of Section 1 and following our subsequent procedure.

We wish to thank our colleague, B. Weiss, for several helpful discussions about this work, and the referee for suggesting several improvements in the exposition.

IBM Watson Research Center

Yorktown Heights, New York

1. A. N. Khovanskir, The Application of Continued Fractions and their Generalizations to Problems in Approximation Theory, translated from the Russian, Noordhoff, Groningen, 1963. MR 27 \#6058.

2. C. Lanczos, Applied Analysis, Prentice-Hall, Englewood Cliffs, N. J., 1956. MR 18, 823.

3. T. J. Rivin \& H. S. Shapiro, "A unified approach to certain problems of approximation and minimization," J. Soc. Indust. Appl. Math., v. 9, 1961, pp. 670-699. MR 24 \#A3462.

4. T. J. RivLIN \& B. Weiss, "Lanczos' $\tau$-method and polynomial approximation in the plane," J. Math. Anal. Appl. (To appear.) 